\title{
Heartbeat: impact of suboptimal response to primary prevention
} statin therapy

A key element in primary prevention of cardiovascular disease (CVD) is the use of statin therapy to lower low-density lipoprotein cholesterol (LDL-C) levels in people at increased risk of CVD with many guidelines recommending a fixed statin dose for each estimated risk category. However, the effectiveness of this approach is limited by variations in adherence to therapy plus individual biological and genetic differences. In this issue of Heart, Akyea and colleagues ${ }^{1}$ assessed the LDL-C response to statin therapy in a prospective cohort of over 165000 primary care patients in the UK Clinical Practice Research Datalink. Overall, $>50 \%$ of patients had a suboptimal response to statin therapy with a $<40 \%$ reduction in LDL-C measured at 24 months. The cumulative incidence of CVD events (median follow-up 6.2 years) was higher in the suboptimal responders compared with the group with an optimal response to statin therapy, even after adjusting for baseline LDL-C and age (figure 1).

In the accompanying editorial, Bittencourt and Cesena ${ }^{2}$ summarise the current guideline recommendations for statin therapy in primary prevention and discuss the strengths and limitations of this study. They suggest, 'Although an interindividual response to statins may occur according to the genetic background, most cases where LDL-C response is less than expected are probably due to lack of adherence or persistence to the treatment. Even though statin-associated side effects do prevent statin use in many patients, the nocebo effect, that is, the report of a side effect by the patient actually due to negative expectation is a frequent cause for interrupting statins among the population'. Further, they conclude: 'Effective implementation of guidelines among healthcare practitioners and the general population has been a challenge for a long time. Both physicians and patients should be targets for approaches aiming at improving adherence to guidelines'.

Correspondence to Professor Catherine M Otto, Division of Cardiology, University of Washington, Seattle, WA 98195, USA; cmotto@uw.edu

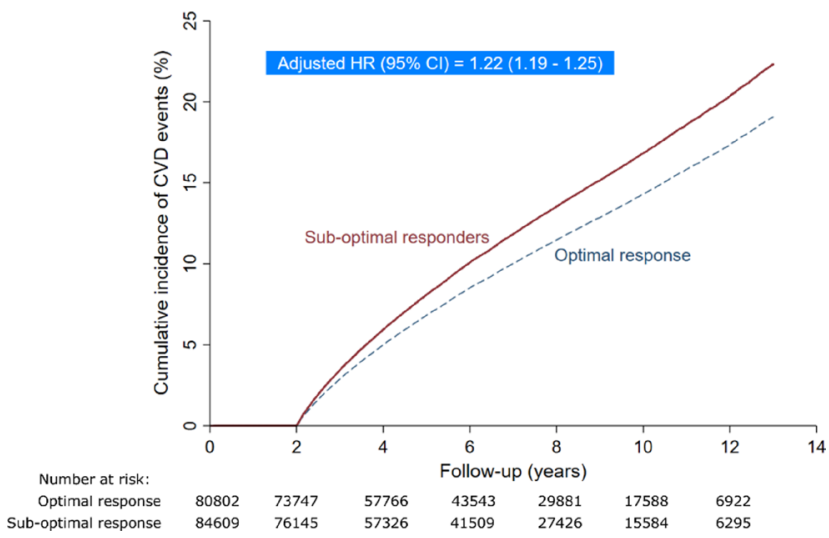

Figure 1 The cumulative incidence curve demonstrated that patients with a suboptimal LDL-C response to statin therapy were associated with a higher risk of CVD events than patients with an optimal response during the follow-up period, with an adjusted HR of 1.22 (95\% Cl: 1.19 to 1.25). Adjusted for age and baseline LDL-c level. CVD, cardiovascular disease; LDL-C, low-density lipoprotein cholesterol.
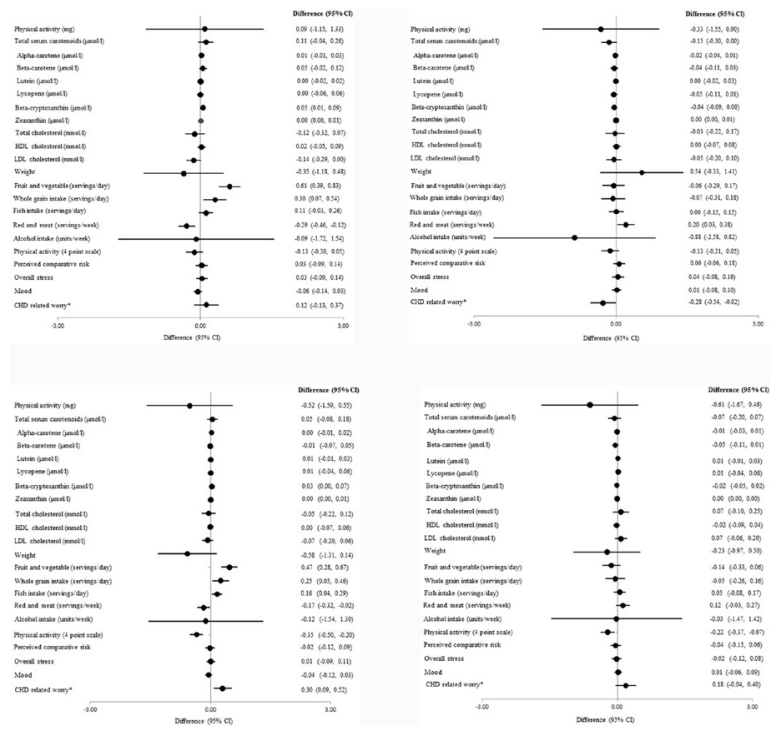

Figure 2 Pairwise comparisons for primary and secondary outcomes-intention-to-treat population (data are reported in natural units). ${ }^{*}$ CHD-related worry only measured at 12-week follow-up; difference, $95 \% \mathrm{Cl}$ and $\mathrm{p}$ value estimated from a linear regression model. Group 1: control; group 2: lifestyle advice only; group 3: phenotypic risk estimate and lifestyle advice; group 4: phenotypic and genetic risk estimates and lifestyle advice. Primary outcome and secondary continuous outcomes: difference and $95 \% \mathrm{Cl}$ estimated from analysis of covariance model with adjustment for baseline. Participants with missing values of the outcome at baseline included using the missing indicator method. CHD, coronary heart disease; HDL, high-density lipoprotein; LDL, low-density lipoprotein.

Another challenging aspect of the primary prevention of CVD is whether communicating the risk of individual phenotypic and genetic factors is effective in positively changing health behaviours. Silarova and colleagues ${ }^{3}$ addressed this question in the Information and Risk Modification (INFORM) trial. In this study, 928 
healthy adults with no known CVD were randomised to four strategies for communicating risk: no information, lifestyle information alone, lifestyle information plus a phenotypic-based risk score and lifestyle information plus a phenotypic and genetic risk score. Based on wrist-worn accelerometer data, there was no effect of any intervention on physical activity or other outcomes, except for self-reported fruit and vegetable intake (figure 2).

These results seem to contradict one of the basi assumptions of precision medicine that patients' behaviours will change in response to individualised risk information. Danchin et $\mathrm{al}^{4}$ point out that any positive effect on lifestyle behaviours might be offset by negative psychological effects-'the psychological consequences of providing precise information on individual risk estimates have not been extensively studied so far, in the field of cardiovascular medicine'. My personal clinical experience also suggests that the time frame of this study ( 3 months) might be too short to measure meaningful change. Patients often need considerably more time both for coming to terms with the risk implications and to implement healthy behaviours. The editorial authors conclude: 'Finally, the way risk is displayed is also crucial. In the INFORM trial, "heart age" (ie, the mean age of individuals with a similar risk) seemed to be more powerful in motivating lifestyle changes than risk estimates displayed as percentages'.

In adults with congenital heart disease (ACHD), standard cardiac serum biomarkers, such as B-natriuretic peptide, are of limited value so that alternate measures for risk stratification are needed. In a study of 590 patients with ACHD, soluble suppressor of tumourigenicity-2 (sST2), which is upregulated in response to myocardial stress, was associated with an increased risk of the primary endpoint of any cardiovascular events over a mean

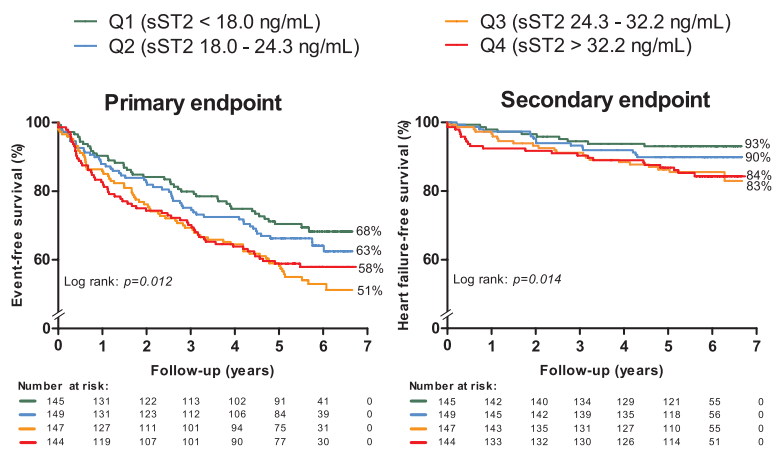

Figure 3 Survival regarding the primary endpoint (any cardiovascular event) and the secondary endpoint (death or heart failure) stratified according to the quartile distribution of sST2. Q1, quartile 1; Q2, quartile 2; Q3, quartile 3; Q4, quartile; SST2, soluble suppression of tumourigenicity-2.
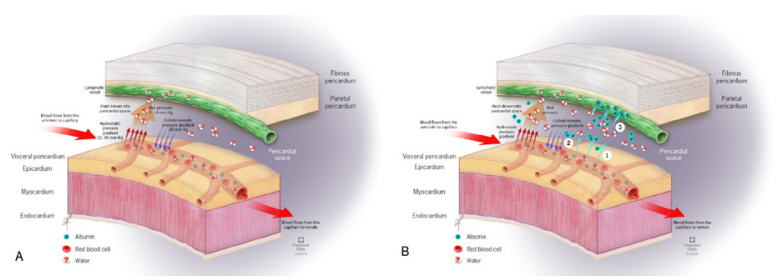

Figure 4 Mechanism of physiological and hypothyroid pericardial effusion formation. (A) Mechanism of physiological pericardial fluid formation in a normal state: the hydrostatic pressure at the arterial end of the capillary is higher than the one at its venous end. Yet, the colloid osmotic pressure generated mainly by plasma proteins is essentially the same at both ends. This results in the filtration of fluids at the arterial end and reabsorption of the fluids at the venous end. Some of the fluid will be channeled towards the lymphatic vessels. (B) Hypothetical mechanism of pericardial effusion formation in hypothyroidism: there is an increase in the vascular permeability to albumin (1), raising the interstitial colloid pressure and hence decreasing the colloid osmotic pressure gradient between the interstitium and the intravascular space (2). This results in a decrease of fluid return to the capillaries. However, there is a decrease in the lymphatic drainage of albumin (3) worsening the gradient mentioned above (2) and reducing the fluid return.

follow-up of 5.8 years ${ }^{5}$ (figure 3 ). In the editorial $^{6}$, Tutarel points out that most patients in this study were asymptomatic and did not have elevated sST2 levels. Perhaps more conclusive results would be seen in a study group with a wider range of disease severity.

The Education in Heart article in this issue reviews the use of stress echocardiography in adults with valvular heart disease. ${ }^{7}$

Pericardial disease in patients with hypothyroidism is the subject of a review article in this issue of Heart. Up to one-third of adults with hypothyroidism have a pericardial effusion and tamponade can occur in severe cases. This article reviews the mechanism, clinical presentation and management of this condition ${ }^{8}$ (figure 4).

The Cardiology in Focus article provides concise guidance on how to succeed in the European Examination in Cardiology including recommendations on how programmes can support cardiology trainees and a list of resources trainees can use in preparing for the examination. ${ }^{9}$

\section{Competing interests None declared.}

\section{Patient consent for publication Not required.}

Provenance and peer review Commissioned; internally peer reviewed.

(C) Author(s) (or their employer(s)) 2019. No commercial re-use. See rights and permissions. Published by BMJ.

\section{(D) Check for updates}

To cite Otto CM. Heart 2019;105:967-968.

Received 21 May 2019

Accepted 21 May 2019

Heart 2019;105:967-968.

doi:10.1136/heartjnl-2019-315446

\section{REFERENCES}

1 Akyea RK, Kai J, Qureshi N, et al. Sub-optimal cholesterol response to initiation of statins and future risk of cardiovascular disease. Heart 2019;53:975-81.

2 Bittencourt MS, Cesena FHY. Statin dose in primary prevention: aim for the target!. Heart 2019;53:969-71.

3 Silarova B, Sharp S, Usher-Smith JA, et al. Effect of communicating phenotypic and genetic risk of coronary heart disease alongside web-based lifestyle advice: the INFORM Randomised Controlled Trial. Heart 2019:53:982-9.

4 Danchin N, Lahlou-Laforet K, Lemogne C. Informing on individual cardiovascular risk: from wishful thinking to hard facts. Heart 2019;53:973-4.

5 Geenen LW, Baggen VJM, van den Bosch AE, et al. Prognostic value of soluble ST2 in adults with congenital heart disease. Heart 2019;53:999-1006.

6 Tutarel 0. sST2: a new kid on the block for patients with ACHD. Heart 2019;53:972.

7 Khattar RS, Senior R. Stress echocardiography in the assessment of native valve disease. Heart 2019;53:1034-43.

8 Chahine J, Ala CK, Gentry JL, et al. Pericardial diseases in patients with hypothyroidism. Heart 2019;53:1027-33.

9 Ooues G, Plummer C, Hall J, et al. How to succeed in the EEGC: a guide for trainees and their trainers. Heart 2019;53:1044-5. 